\title{
Commentary: "Up" within "Down"
}

\author{
ROBERT S. HATTEN [1] \\ The University of Texas
}

\begin{abstract}
This commentary considers ways in which meter implies a virtual environmental field, one that has close correlations with dancers' actual experiences of movement within gravitational constraints, despite their verbal descriptions that might suggest the contrary. Upward movements on downbeats are interpretable as virtual agents' expenditures of energy against virtual gravitational constraints, akin to those dancers experience in ballet. Thus, "up" as movement can imply "down" as gravitational field.
\end{abstract}

Submitted 2014 October 10; accepted 2014 November 3.

KEYWORDS: music, meter, ballet, virtual environment, virtual agency, gravity

THE issue of virtual embodiment in our experience of meter is given a strong test in Jonathan Still's superbly researched article. In my commentary, I will focus on a single point that has bearing on his conclusion that my theory of a virtual musical environment, featuring a musical force akin to gravity (according to Steve Larson), may not capture the embodied experience of dancers, as opposed to instrumentalists, when it comes to meter. Specifically, I suggested that we hear meter as a framework in which we experience a virtual environment (Hatten, 2004, p. 117), and following Larson (2012, pp. 148149), the downbeat is experienced as the platform toward which gravity pulls a virtual body. But Still points out, quite reasonably, that dancers often move upward instead of downward on a metric downbeat. In a recent article (Hatten, 2012), I argue that upward leaps to a downbeat (and other events) in instrumental music can counter the musical forces implied by meter (and tonality), and thereby provide evidence of a virtual agent's energies within a virtual environment. Thus, merely going up need not negate the potential experience of a virtual field in which we orient ourselves gravitationally with respect to the platforms of metric downbeats (and tonics). Rather, upward motion may in fact emphasize the independent agency of a virtual body that can expend sufficient energy to overcome gravity.

Turning to actual bodies, the experience of going up on the downbeat has similar dynamics: one presses energetically against the firm surface (gravitational platform) of a floor or stage, acknowledging gravity in a very direct sense, in order to move upward, even on a downbeat. There are various movement possibilities, one of which (mentioned in a footnote Still cites from Hatten, 2004, p. 304) gives the apparently counterintuitive feel of sinking down on an upbeat (plié) to generate the energy that then transfers into an upward lift on the downbeat (elevé, or more precisely, relevé, if the rise is directly from the plié). A similar movement, although the energetic springing up must be accomplished on the beat itself, is the elevé on a downbeat without a prior plié. In either case, there is a "press-down-to-spring-up" movement (of varying velocity) occurring on the downbeat itself, wherein most of the duration is felt as "up," despite the quick springing motion of having pressed down against the platform simultaneously with the downbeat. I would argue, however, that even if the body were to move down on an upbeat to gather energy for the spring prior to the downbeat, the dynamics of the gravitational field would not be changed. Instead, an experience of "lift" as earned through agential effort is fostered, in each of the situations described. And the sense of embodied lift depends on our experience of gravity that is "escaped"-however briefly - by an individual's expenditure of energy (whether actual or virtual). The aesthetics of this kind of lift are fundamental to classical ballet in the $19^{\text {th }}$ century, and gravitational escape (often by a literal lift or leap) is in some sense understood with respect to an individual's willful independence-not merely bodily (interpretable simply as feeling the freedom to move, or a delight in energetic movement), but also spiritually (interpretable more allegorically as an experience of metaphysical freedom or even transcendence of life). By contrast, Nijinsky's stomping choreography for Stravinsky's Rite of Spring enraged members of the audience in Paris in 1913 because it denied classical ballet's allegories of 
transcendence and substituted instead a primitivistic view of the body (all downbeats, in effect-as suggested almost literally by the consecutive down-bows in the strings for reiterations of the famous Rite chord).

Thus, even counterintuitive movements ("up" on a downbeat) depend on a gravitational field (both actual and virtual) for their very meaning. The problem then becomes: why is this so difficult to teach dancers? Here, Still is clearly better positioned to discover pedagogical answers, but I might suggest that part of the problem involves the disconnect between language and embodiment, in which respondents may not be aware that "up" can imply "down"- "up" with reference to movement, "down" with reference to the force field by which movement is constrained, against which energy must be expended in order to rise, and with reference to which any rise is given meaning.

A problem not addressed by Still is how such a spatial analogue as a gravitational field could emerge as a stable interpretation from the temporal experience of meter (which is constantly cycling through periodicities of "down" vs. "up"). I have considered the possibility that constant recycling may serve to continually "refresh" the virtual environmental force field for perception - perhaps not unlike the way sequential saccades of the eye serve to refresh one's field of vision (although focusing on various objects in that field may be the primary motivation). But perhaps the most obvious answer is that meter arises from periodic motions of the kind that humans first embodied in dance. The debt to dance is palpable.

I would like to thank Jonathan Still for his probing article, and for the invitation of the editor to respond. I look forward to following the development of Still's ideas, especially his application of the related notions of empathy and perspective-taking to his teaching of meter to dancers.

\section{NOTES}

[1] Correspondence can be addressed to: Prof. Robert S. Hatten, Butler School of Music, The University of Texas at Austin, 1 University Station E3100, Austin, Texas 78712-0435, rohatten@austin.utexas.edu.

\section{REFERENCES}

Hatten, R. S. (2004). Interpreting musical gestures, topics, and tropes: Mozart, Beethoven, Schubert. Bloomington and Indianapolis: Indiana University Press.

Hatten, R. S. (2012). Musical forces and agential energies: An expansion of Steve Larson's model. Music Theory Online, 18(3). Retrieved from: http://mtosmt.org/issues/mto.12.18.3.hatten.php

Larson, S. (2012). Musical forces: Motion, metaphor and meaning in music. Bloomington and Indianapolis: Indiana University Press.

Still, J. (2015). How down is a downbeat? Feeling meter and gravity in music and dance. Empirical Musicology Review, 10(2), 121-134. 